\title{
Repeated placement of minocycline microspheres gives small additional probing depth reductions in periodontal therapy
}

\section{In patients with moderate to advanced periodontal disease, is scaling plus minocycline microspheres more effective than scaling alone?}

\section{Williams RC, Paquette DW, Offenbacher S, Adams DF, \\ Armitage GC, Bray K, et al. Treatment of periodontitis by local administration of minocycline microspheres: a controlled trial. Periodontol 2001; 72:1535-1544}

Design In this 9-month multicentre, controlled trial, 748 patients with moderate to advanced periodontitis were randomised into one of three parallel groups: (1) control group (scaling alone), (2) scaling plus vehicle and (3) scaling plus minocycline microspheres. Only sites initially $5 \mathrm{~mm}$ or deeper were included in the statistical analysis.

Intervention At baseline all patients received scaling. At baseline, 3 and 6 months placebo or minocycline microsphere groups received placebo (vehicle) or minocycline microspheres to sites with probing depth $\geq 5 \mathrm{~mm}$.

Outcome measures Probing depth (PD) measurements were taken at baseline, 1, 3, 6 and 9 months and treatment outcome was assessed as changes in PD.

Results At 9 months a significant reduction in mean PD was noted in all three groups of $\sim 1 \mathrm{~mm}$. When compared to scaling, the minocycline microspheres group displayed a significantly greater reduction in probing depth of $\sim 0.3 \mathrm{~mm}$. This difference remained when looking into predetermined subgroups such as age, gender and smoking status.

Conclusion Extrapolation from Table 2 in the Williams et al article indicates an NNT of 9. Thus, one would need to treat nine sites for one site to obtain an additional $\sim 0.3 \mathrm{~mm}$ pocket depth reduction.

\section{Commentary}

Nonsurgical periodontal therapy is based on information/motivation, instruction in proper oral hygiene and meticulous supra and subgingival scaling and root planing (S\&RP). Treatment outcomes are evaluated after 3-6 months as full mouth plaque score (FMPS \%) to assess patient compliance, full mouth bleeding on probing scores (FMBS \%); probing depth reduction and gain of clinical attachment (CAL). Patient-related supportive care is then designed to maintain treatment outcome. The potential of this treatment modality has been extensively reviewed by Cobb. ${ }^{1,2}$ In the last 15 years, a number of studies on the adjunctive effect of locally applied antimicrobial agents have been presented, the outcomes of which are not conclusive.

This randomised controlled clinical trial has three parallel groups with around 250 patients in each group. All three groups had a baseline S\&RP. This report of the study does not indicate whether randomisation was carried out before or after S\&RP or whether treatment and examination were carried out by the same clinician. The first would reduce bias, and the second raises questions regarding the blindness of the study. However, the authors have

Address for correspondence: Dr Ray C Williams, Department of Periodontology UNC School of Dentistry, CB 7450, Chapel Hill, NC 27599-7450. E-mail: ray_williams@ dentistry.unc.edu confirmed that randomisation was performed prior to S/RP and that treatment and examination were performed by separate individuals. Following baseline therapy the test group was retreated with minocycline microspheres at 3 and 6 months, whereas the pure control group (S\&RP only) was not retreated during the 9-month period. Thus there were two more interventions in the test group.

$\mathrm{PD}$ reduction at 9 months was used as the primary efficacy variable. Plaque scores were not given although the importance of patient compliance in supragingival plaque control (with impact also on the subgingival microflora) is well documented. Probing depth reduction with S\&RP plus minocycline microspheres was statistically greater than with S\&RP alone (1.32 vs $1.08 \mathrm{~mm})$. With group sizes as in the present study, statistical significance can always be achieved; however, the clinical significance of the difference can be questioned.

In addition the PD reduction following S\&RP alone was less than demonstrated in several studies (eg ${ }^{3-5}$ ) or as reviewed by Cobb $^{1,2}$ especially for pockets $6 \mathrm{~mm}$ and deeper, which may question the quality of the basic therapy itself. PD reduction of $\geq 2 \mathrm{~mm}$ was achieved in $32.9 \%$ of "S\&RP-alone" sites only as compared to $40.5 \%$ following S\&RP + minocycline microspheres. The small reduction in bleeding scores for sites $6 \mathrm{~mm}$ and deeper further supports this suspicion. In summary, this study does not convincingly support the benefit of using minocycline microspheres as an adjunct measure in basic periodontal therapy.

\section{Practice point}

- The study shows a statistical benefit to microsphere use, but further studies would be needed to show convincing clinical benefit.

\section{Lars Laurell}

Eastman Dental Institute for Oral Health Care Sciences, University College, London, UK

1. Cobb CM. Clinical significance of non-surgical periodontal therapy: an evidence based perspective of scaling and root planing. J Clin Periodontol 2002; (Supplement 2):6-16.

2. Cobb CM. Non-surgical pocket therapy: mechanical. Ann Periodontol 1996; $1: 443-490$

3. Kaldahl WB, Kalkwarf KL, Patil KD, Molvar MP, Dyer JK. Long-term evaluation of periodontal therapy: response to 4 therapeutic modalities. J Periodontol 1996; 67:93-102.

4. Ramfjord SP, Caffesse RG, Morrison EC. 4 modalities of periodontal treatment compared over 5 years. J Clin Periodontol 1987; 14:445-452.

5. Badersten A, Nilveus R, Egelberg J. Effect of non-surgical periodontal therapy. II. Severely advanced periodontitis. J Clin Periodontol 1984; 11:63-76.

Evidence-Based Dentistry (2003) 4, 5.

doi:10.1038/sj.ebd.6400161 